\title{
Article \\ Polyvinyl-Pyrrolidone-Coated Silver Nanoparticles-The Colloidal, Chemical, and Biological Consequences of Steric Stabilization under Biorelevant Conditions
}

\author{
Andrea Rónavári ${ }^{1,+}(\mathbb{D})$, Péter Bélteky ${ }^{1,+} \mathbb{D}^{\mathbb{D}}$, Eszter Boka ${ }^{1}$, Dalma Zakupszky ${ }^{1}$, Nóra Igaz ${ }^{2} \mathbb{D}$, Bettina Szerencsés $^{3}$, \\ Ilona Pfeiffer ${ }^{3}\left(\mathbb{D}\right.$, Zoltán Kónya ${ }^{1,4, * \mathbb{D}}$ and Mónika Kiricsi ${ }^{2}$
}

\section{check for}

updates

Citation: Rónavári, A.; Bélteky, P.; Boka, E.; Zakupszky, D.; Igaz, N.; Szerencsés, B.; Pfeiffer, I.; Kónya, Z.; Kiricsi, M. Polyvinyl-Pyrrolidone -Coated Silver Nanoparticles-The Colloidal, Chemical, and Biological Consequences of Steric Stabilization under Biorelevant Conditions. Int. J Mol. Sci. 2021, 22, 8673. https:// doi.org/10.3390/ijms22168673

Academic Editor: Pavel Rossner

Received: 19 July 2021

Accepted: 9 August 2021

Published: 12 August 2021

Publisher's Note: MDPI stays neutral with regard to jurisdictional claims in published maps and institutional affiliations.

Copyright: (c) 2021 by the authors. Licensee MDPI, Basel, Switzerland. This article is an open access article distributed under the terms and conditions of the Creative Commons Attribution (CC BY) license (https:/ / creativecommons.org/licenses/by/ $4.0 /)$.
1 Department of Applied and Environmental Chemistry, Faculty of Science and Informatics, University of Szeged, H-6720 Szeged, Hungary; ronavari@chem.u-szeged.hu (A.R.); peti0225@gmail.com (P.B.); bokaeszti@gmail.com (E.B.); z.dalma.ballet@gmail.com (D.Z.)

2 Department of Biochemistry and Molecular Biology, Faculty of Science and Informatics, University of Szeged, H-6726 Szeged, Hungary; noraigaz@gmail.com (N.I.); kiricsim@gmail.com (M.K.)

3 Department of Microbiology, Faculty of Science and Informatics, University of Szeged, H-6726 Szeged, Hungary; betti414@gmail.com (B.S.); pfeiffer@bio.u-szeged.hu (I.P.)

4 MTA-SZTE Reaction Kinetics and Surface Chemistry Research Group, H-6720 Szeged, Hungary

* Correspondence: konya@chem.u-szeged.hu; Tel.: +36-(62)-544620

$+\quad$ These authors contributed to the research equally.

\begin{abstract}
Background: Several properties of silver nanoparticles (AgNPs), such as cytotoxic, anticancer, and antimicrobial activities, have been subjects of intense research; however, important aspects such as nanoparticle aggregation are generally neglected, although a decline in colloidal stability leads to a loss of the desired biological activities. Colloidal stability is affected by $\mathrm{pH}$, ionic strength, or a plethora of biomolecules that interact with AgNPs under biorelevant conditions. (2) Methods: As only a few studies have focused on the relationship between aggregation behavior and the biological properties of AgNPs, here, we have systematically evaluated this issue by completing a thorough analysis of sterically (via polyvinyl-pyrrolidone (PVP)) stabilized AgNPs that were subjected to different circumstances. We assessed ultraviolet-visible light absorption, dynamic light scattering, zeta potential measurements, in vitro cell viability, and microdilution assays to screen both colloidal stability as well as bioactivity. (3) Results: The results revealed that although PVP provided outstanding biorelevant colloidal stability, the chemical stability of AgNPs could not be maintained completely with this capping material. (4) Conclusion: These unexpected findings led to the realization that stabilizing materials have more profound importance in association with biorelevant applications of nanomaterials than just being simple colloidal stabilizers.
\end{abstract}

Keywords: chemical stability; steric stabilization; aggregation behavior; antimicrobial activity; cytotoxicity; anticancer activity

\section{Introduction}

Due to the numerous favorable features of nanomaterials, their application in various fields, including medical, food, consumer, health care, and industrial purposes, is constantly increasing. However, characterization of their chemical and biological behavior is fundamental to ensure safe and predictable application, especially upon encountering living organisms. In recent years, the design and fabrication of next-generation silver-based nanomaterials have been subjects of intense research in the field of material sciences [1,2]. Silver nanoparticles (AgNPs) are considered to be one of the most heavily investigated, most frequently synthesized and commercialized nanomaterials owing to their optical, electrical, and thermal features as well as catalytic activities [3,4].

In the biomedical field, the excellent properties of nanosilver are especially exploited since AgNPs are known to exhibit outstanding antimicrobial, anti-inflammatory, anticancer, 
and antiangiogenic activities. These unique chemical, physical, and biological properties of AgNPs are massively influenced by a number of factors such as nanoparticle size and morphology or by surface coating, which are generally determined at nanoparticle synthesis [5-7]. Therefore, proper selection of the synthesis method is crucial for achieving the desired particle properties for specific applications $[8,9]$. In the last decade, a plethora of procedures have been developed for AgNP production; however, the most common approach, which is also highly effective and easy to control, is the chemical reduction of a silver salt using various reducing and capping agents [10].

Employment of nanoparticles for any function requires nanoparticles of reliable longterm stability, with controlled and well-defined properties. In fact, colloidal stability and aggregation propensity are issues that should be more profoundly regarded. Aggregation might cause a substantial decrease in the effective surface area of nanomaterials, which can lead to the reduction or complete loss of the advantageous nano-properties [11]. As the synthesis method might also govern the lasting stability of silver nanoparticle suspensions, numerous chemical and biological approaches have been adopted and optimized (reaction circumstances, reducing and stabilizing agents) to improve the parameters influencing the stability of silver nanoparticles [12,13]. Different types of capping and stabilizing agents, such as surfactants (e.g., cetrimonium bromide (CTAB)), polymers (e.g., polyvinyl pyrrolidone (PVP)), and green materials (such as extracts of plant parts, e.g., tea leaves or coffee beans) have been utilized for the surface modification of AgNPs to prevent aggregation [14-16]. Various stabilization mechanisms such as electrostatic, steric, and electrosteric stabilization were engaged upon synthesis, yielding nanoparticles with different physical, chemical, and biological characteristics and colloidal stability [17]. The colloidal stability of nanoparticles designed for therapeutic utilization is absolutely essential since nanoparticle aggregation in living systems, under biorelevant conditions, can not only directly determine the biological (such as antimicrobial, cytotoxic, or drug delivery) activity of the particles, but it can also have life-threatening consequences [15]. It is therefore intriguing that although plenty of studies have reported how the various reducing and stabilizing entities affect the size, size distribution, and shape as well as the biological activity of AgNPs, nevertheless, the scientific literature is largely lacking in data on the aggregation behavior of nanoparticles, especially the effects that particle aggregation exerts on the bioactive features of the produced nanomaterials.

In our recently published papers, we described environmentally benign synthesis approaches to AgNP formation, and we also showed that the various green materials used for stabilization and reduction of metal ions have a defining role in determining the colloidal stability and biological activity of the obtained green nanoparticles $[8,18]$. The effect of particle size on the aggregation behavior of different AgNP suspensions and the modulation of antimicrobial activity and toxicity as the function of nanoparticle aggregation were also investigated. Thereafter, we systematically analyzed and compared the aggregation behavior of electrosterically and electrostatically stabilized AgNPs in different biological systems using an experimental procedure consisting of a series of chemical and in vitro biological measurements [19]. We demonstrated that the electrostatically stabilized AgNPs completely lost their biological activity due to aggregation, while the electrosterically stabilized AgNPs were less prone to aggregate under biorelevant experimental conditions. Through these experiments, we highlighted the need for careful selection of the proper nanomaterial synthesis method to achieve the desired particle properties and raised awareness for a compulsory comprehensive chemical and biological screening procedure to assess the stability and aggregation propensity of nanoparticles to estimate their fate and behavior under biorelevant conditions prior to their application. However, sterically stabilized silver nanoparticles have never been involved in a systematic evaluation of the aggregation-biological activity relationship; therefore, the present paper aims to fill that void in order to provide a complete view on the possible nanoparticle stabilization types regarding this issue. For this purpose, the most common steric stabilizer, the natural biopolymer PVP, was used to produce silver nanoparticles. The obtained particles were ex- 
amined thoroughly under biorelevant conditions to delineate the effects of varying $\mathrm{pH}$ and differing concentrations of sodium chloride, glucose, glutamine, and fetal bovine serum (FBS) on particle stability. Moreover, the aggregation behavior of these PVP-stabilized particles was measured in a time-dependent manner to evaluate the nanoparticle aggregation grade and its impact on cytotoxicity and antimicrobial activity in the function of time. This experimental setup provided further evidence on the profound significance of the degree of silver nanoparticle aggregation in modulating biological activity against cancerous and non-cancerous human cells as well as various microbes and how this feature could be affected, in particular, by steric stabilization.

\section{Results and Discussion}

\subsection{Nanoparticle Morphology and Crystallinity}

The morphological properties of the synthesized AgNPs are presented in Figure 1 with the corresponding TEM images, electron diffraction patterns, and their respective particle size distribution histograms. Both PVP-utilizing reactions resulted in spherical nanoparticles. The smaller molecular weight (MW) PVP-stabilized system yielded larger particles, around $12 \mathrm{~nm}$, while the larger molecular weight system generated smaller particles of about $6 \mathrm{~nm}$ diameter in accordance with the relevant literature. In their contribution, Madkour et al. proposed that during nanoparticle synthesis, one end of the stabilizing polymers may attach to the forming particles, while the rest of the chain forms a loose adsorbate layer around them [20]. The degree of coverage around the particles increases with polymer molecular weight, thus inhibiting further $\mathrm{Ag}^{+}$reduction on the surface to a higher degree, resulting in smaller particles compared to systems utilizing smaller MW stabilizers, where the particles have a larger free surface, rendering particle growth possible. The sample prepared with 40k PVP demonstrated prominent DebyeScherrer rings, corresponding to 2.35 and $1.44 \AA$ lattice distances, or Miller indices of (111) and (220), respectively; to a smaller degree, rings corresponding to (220) and (311) were detected, verifying the chemical composition of the particles $[8,18,21,22]$. AgNPs synthesized by $50 \mathrm{k}$ PVP also demonstrated these rings, although in a much more diffuse manner, due to their smaller particle size. Furthermore, an additional ring appeared, which, according to the Inorganic Crystal Structure Database (ICSD), corresponds to the (202) crystal facet of silver oxide $(2.73 \AA)$, demonstrating the somewhat reduced chemical uniformity of silver nanoparticles stabilized through 50k PVP [23].

\subsection{Chemical Stability Analysis}

According to the literature and our previous results, the characteristic surface plasmon peaks of silver nanoparticles can undergo various changes due to interfering agents in the liquid media $[16,18,24]$. Their $\lambda_{\max }$ may redshift due to surface interactions, and their absorbance intensities can decrease with aggregation $[25,26]$. However, the results in Figure 2, describing the effect of $\mathrm{NaCl}$ on silver nanoparticles stabilized by various concentrations of PVP, cannot be explained by the above-mentioned phenomena. Each spectrum, corresponding to AgNP samples mixed with $100 \mathrm{mM} \mathrm{NaCl}$, demonstrated slight SPR blue shifts and absorbance increases at the local minima around $325 \mathrm{~nm}$ compared to the untreated AgNP samples. Blue shifts may occur due to a decrease in nanoparticle diameter, while the intensity increase of the absorbance valley can be explained by the formation of $\mathrm{AgCl}$ precipitation [24,27].

The UV-Vis results implied that all measured samples are prone to decomposition upon surface contact with $\mathrm{Cl}^{-}$ions through $\mathrm{AgCl}$ formation, although to varying degrees. As these experiments were aimed at aiding in the selection of the most proper AgNP@PVP system, to be examined in the subsequent part of the study, according to these findings, the sample with the smallest blue shift accompanied by the largest absorbance intensity difference between the $325 \mathrm{~nm}$ valley and the SPR peak was chosen. Smaller molecular weight PVP proved to be more favorable for AgNP stabilization as smaller polymer chains in the same mass result in a larger number of single chains, which can spread out more 
evenly on the nanoparticle surfaces [28,29]. Gaining better PVP coverage by increasing the PVP concentration (from 2 to $10 \mathrm{mg} / \mathrm{mL}$ ) proved to be ineffective as increasing the amount of PVP most likely pushed its concentration beyond a critical point, where polymerpolymer interactions became more prominent than polymer-nanoparticle interactions, leading to the reduced chemical stability of AgNPs in an environment containing large amounts of chlorine (AgNP samples mixed with $100 \mathrm{mM} \mathrm{NaCl}$ ). The best results were produced with PVP of 40k average MW, applied in a concentration of $2 \mathrm{mg} / \mathrm{mL}$. As this sample demonstrated better chemical uniformity as well, it was labeled AgNP@PVP and used in later experiments, and subsequent references to this abbreviation will always correspond to the sample stabilized by $2 \mathrm{mg} / \mathrm{mL}$ (13.3 mg/mg AgNP) 40k MW PVP.

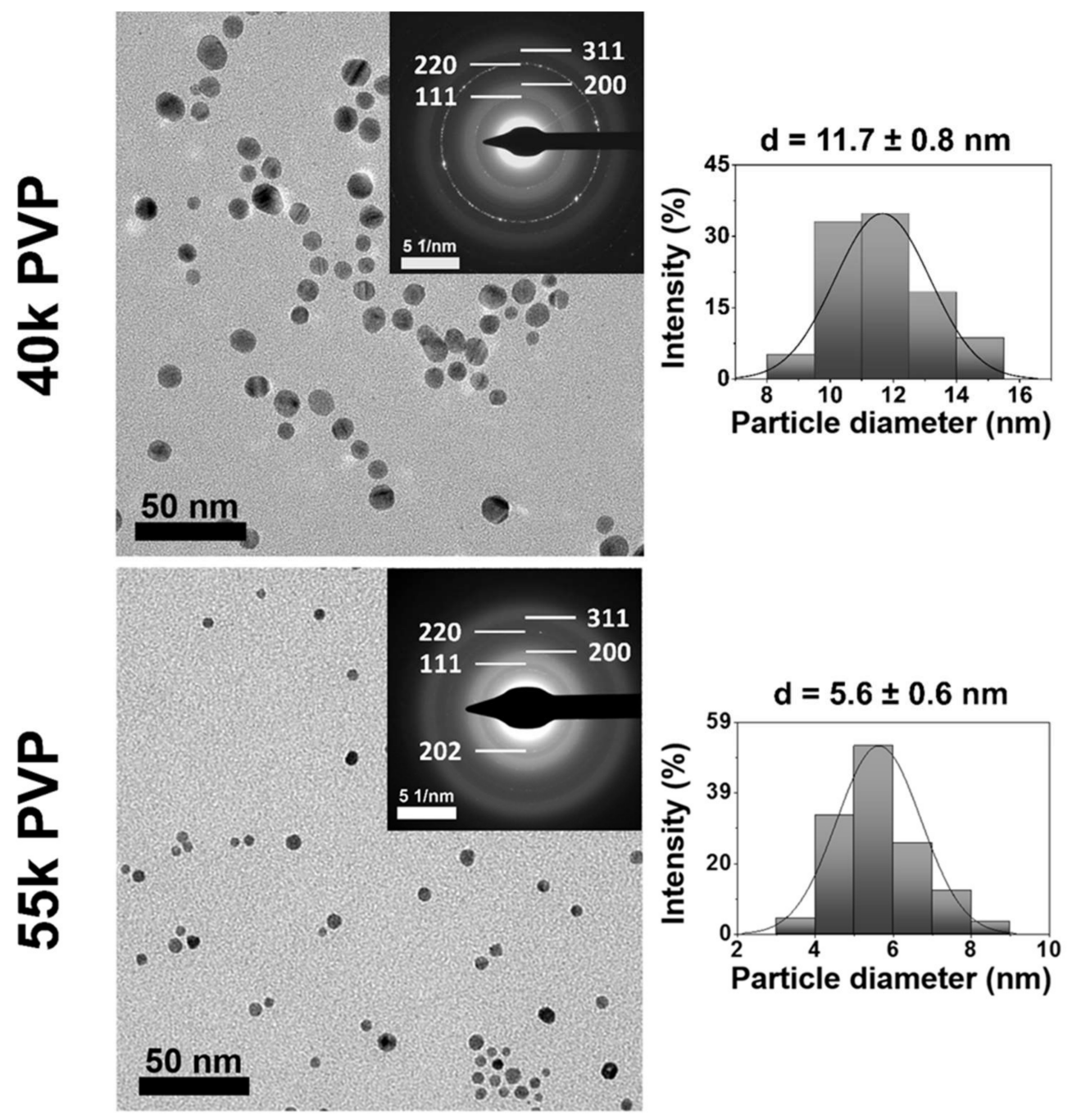

Figure 1. Transmission electron microscopic (TEM) images, electron diffraction (ED) patterns, and size distribution histograms of silver nanoparticles stabilized by 40k (top) and 55k (bottom) molecular weight PVP. 


$-\mathrm{AgNP}$
$-\mathrm{AgNP}+\mathrm{NaCl}$
$-\mathrm{AgCl}$

CPVP $_{\text {P }}$

$2 \mathrm{mg} / \mathrm{mL}$
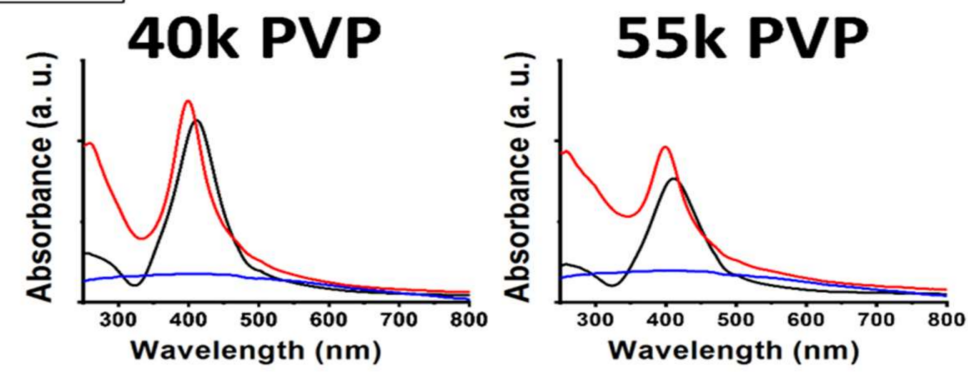

$4 \mathrm{mg} / \mathrm{mL}$
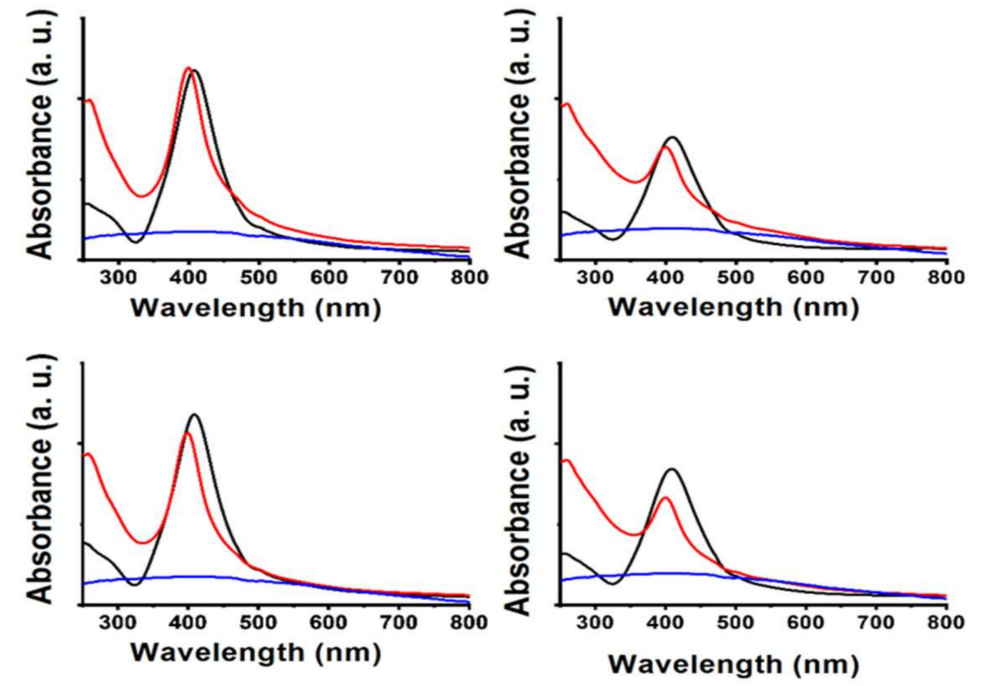

$10 \mathrm{mg} / \mathrm{mL}$

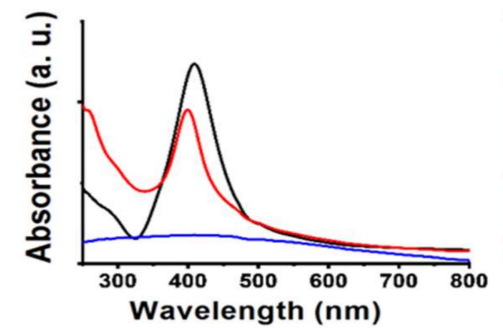

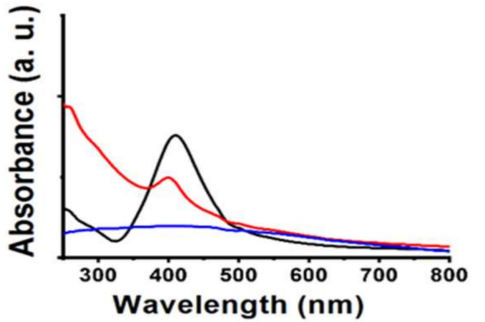

Figure 2. UV-Vis spectra demonstrating the chemical degradation of 40k (left panels) and 55k (right panels) molecular weight PVP-stabilized silver nanoparticles through $\mathrm{AgCl}$ precipitation induced by incubation with $100 \mathrm{mM} \mathrm{NaCl}$ for $20 \mathrm{~h}$ under various PVP concentrations (indicated on the left side of the panels).

\subsection{Aggregation Behavior Assays \\ 2.3.1. The Effect of $\mathrm{pH}$}

Figure 3 demonstrates the effects of $\mathrm{pH}$ on the colloidal stability of AgNP@PVP.

According to the dynamic light scattering (DLS) and zeta potential results, the alkaline condition of $\mathrm{pH} 9$ was the most favorable environment, closely followed by the neutral $\mathrm{pH}$ of 7.2. Under these circumstances, the smallest hydrodynamic diameters were detected, which did not increase substantially throughout the experiments. This finding is important since a significant rise in the hydrodynamic diameter would mean extensive particle aggregation [30,31]. Moreover, the lowest zeta potentials-a marker for high colloidal stability-were measured under $\mathrm{pH} 7.2$ and $\mathrm{pH} 9$ conditions as a result of the increasing $\mathrm{OH}^{-}$concentration of the systems. However, a further decrease in the $\mathrm{pH}$ led to higher Zaverage values under $\mathrm{pH} 5$, which were higher than those obtained in a $\mathrm{pH} 3$ environment. The proposed mechanism explaining these observations is surmised in Scheme 1. 
pH Dependence:

\section{DLS}

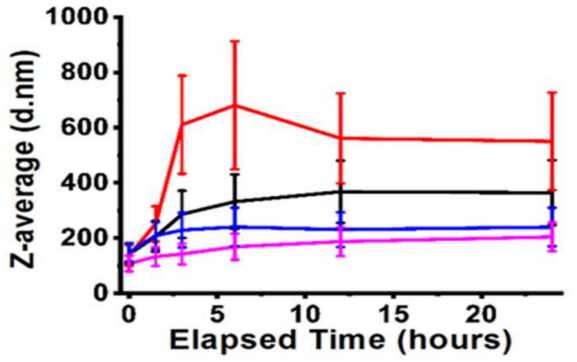

\section{UV-Vis}
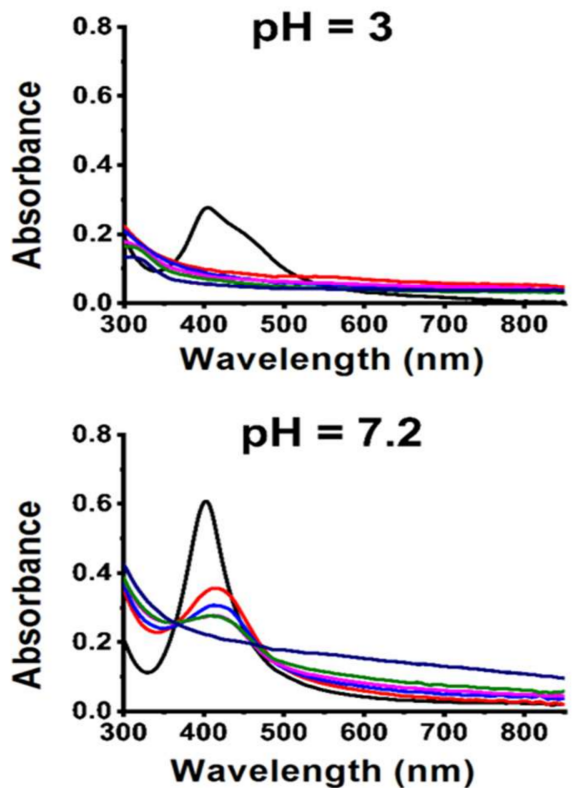

$\mathrm{pH}=3$

$\mathrm{pH}=5$

$\mathrm{pH}=7.2$

$\mathrm{pH}=9$
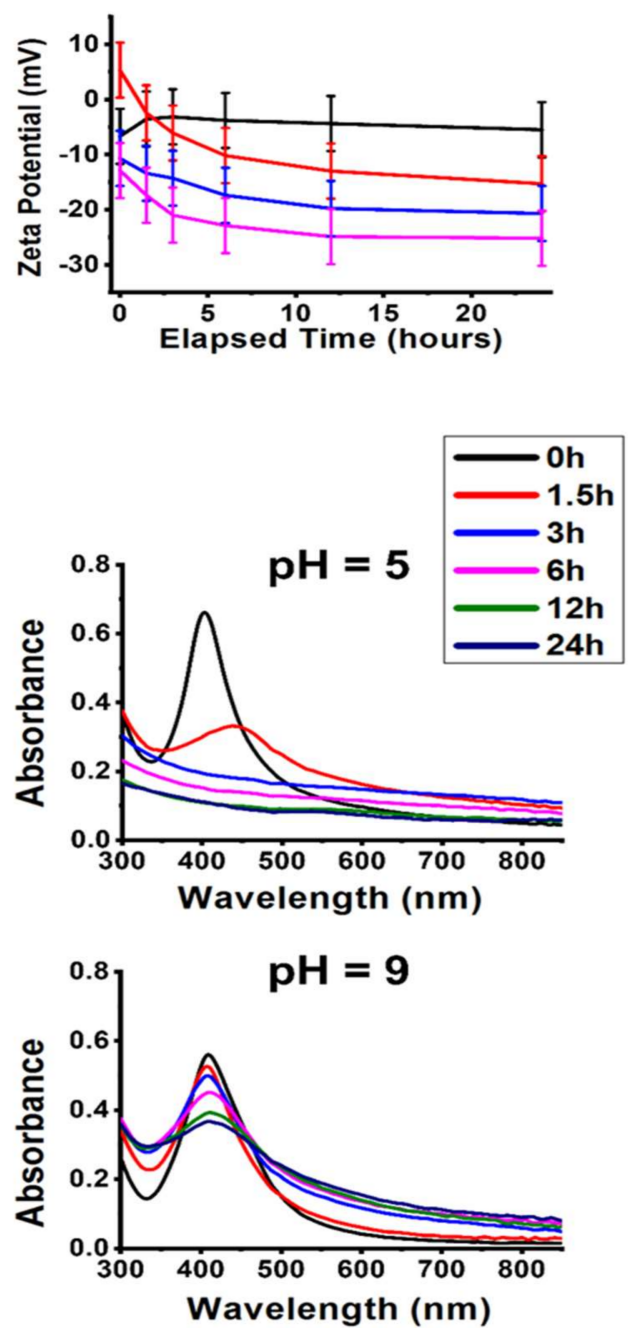

Figure 3. The effect of $\mathrm{pH}$ on the aggregation behavior of PVP-stabilized silver nanoparticles. Dynamic light scattering (DLS) results (top) are represented as average hydrodynamic diameter (Z-average) and zeta potential changes; UV-Vis absorbance spectra (bottom) of PVP-stabilized silver nanoparticles taken over $24 \mathrm{~h}$ under various $\mathrm{pH}$ values. 


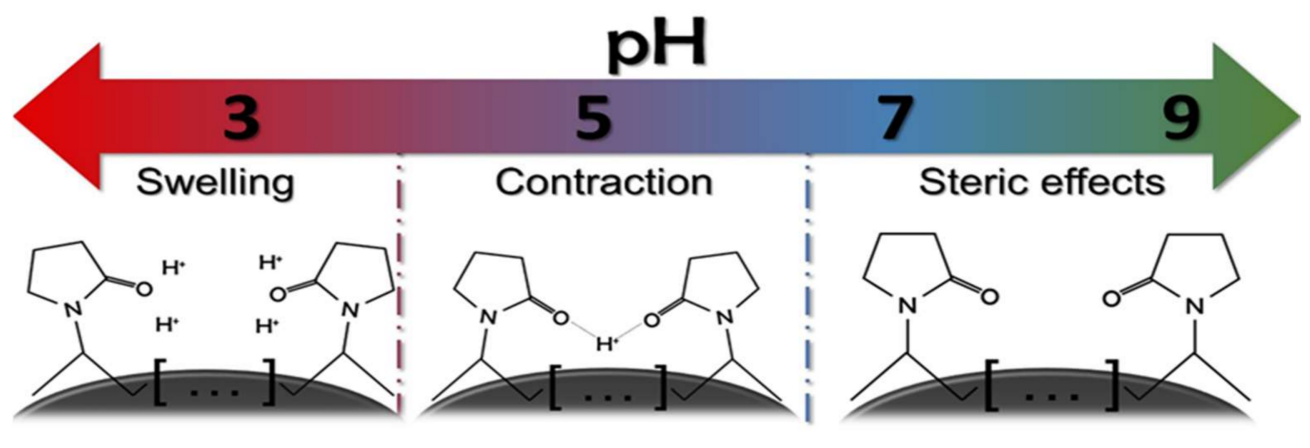

$\mathrm{NaCl}$

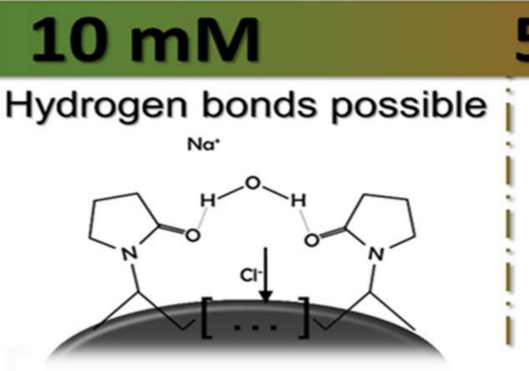

$50 \mathrm{mM}$

$150 \mathrm{mM}$

Chain interactions disrupted

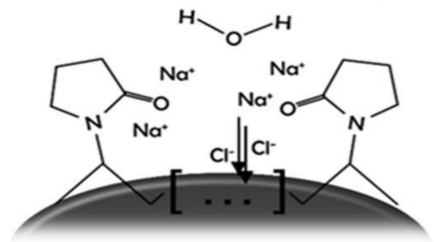

\section{Biomolecules}

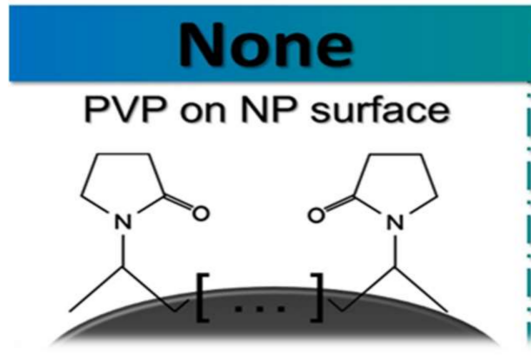

Physiological amount

\section{Additional surface adsorption}

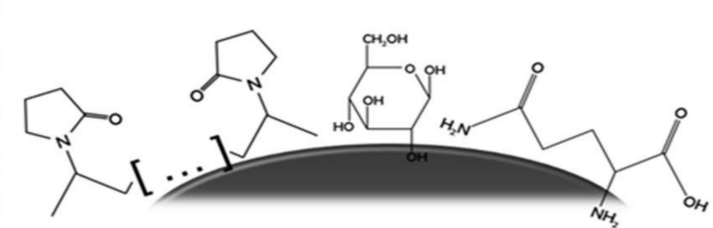

Scheme 1. The effect of environmental conditions on the steric stabilization of silver nanoparticles protected by polyvinyl pyrrolidone.

At $\mathrm{pH} 5$, the moderately increased $\mathrm{H}^{+}$concentration allows the formation of "bridges" between pyrrolidone groups, contracting the AgNP cappings or possibly interconnecting adjacent AgNPs, resulting in an increased aggregation grade [32]. Nevertheless, further increase in the $\mathrm{H}^{+}$concentration of the samples led to the swelling of PVP, in good agreement with the literature [33]. This swelling granted steric stabilization of the nanoparticles, and, therefore, lower average hydrodynamic diameters were detected at this $\mathrm{pH}$.

In our previous publication, changes in the $\mathrm{Z}$-average values were closely accompanied by alterations in the zeta potential, which was understandable, given the fact that the samples investigated there possessed stronger electrostatic characteristics [18]. However, AgNP@PVP is an overwhelmingly sterically stabilized system, and, as such, the interrelationship of hydrodynamic diameter and zeta potential and the changes within are not so straightforwardly linked. Even though the sample corresponding to $\mathrm{pH} 5$ demonstrated the largest aggregates, the highest zeta values were detected at $\mathrm{pH} 3$. The other three conditions all facilitated decreasing zeta potential tendencies. These observations can be explained by the gradual formation of $\mathrm{AgCl}$ precipitation on the surface of the nanoparticles, which is 
known to decrease the overall zeta potential, induced by the presence of the $10 \mathrm{mM} \mathrm{NaCl}$ background electrolyte concentration [30,34].

The DLS and zeta potential results are in close correlation with the UV-Vis spectra shown in Figure 3. Aggregation can be interpreted from the redshift and intensity decrease of the characteristic SPR absorbance peaks of AgNPs, while the formation of $\mathrm{AgCl}$ is once again demonstrated by the absorbance increase of the local minima before the SPR peaks (around $325 \mathrm{~nm}$ ). Slight nanoparticle aggregation was observed in all samples, with increasing intensity towards acidic $\mathrm{pH}$. Interestingly, a very steep SPR decline is observed on the spectra obtained at $\mathrm{pH} 3$, although the spectral baselines did not increase. Thus, this feature is most likely due to the formation of compact aggregates rather than chemical decomposition through $\mathrm{AgCl}$. This explains why the zeta potential of this system trended in the opposite direction compared to the rest of the $\mathrm{pH}$-related experiments.

In summary, the colloidal stability of AgNPs stabilized by PVP remained largely unaffected on alkaline $\mathrm{pH}$ values, while mildly acidic $\mathrm{pH}$ caused notable aggregation due to the formation of $\mathrm{H}^{+}$bridges among pyrrolidone groups. However, the chemical stability of the particles was not secured through steric stabilization, and the chemical decomposition of $\mathrm{AgNPs}$ via $\mathrm{AgCl}$ precipitation was noticeable.

\subsubsection{The Effect of $\mathrm{NaCl}$}

The impact of physiological $\mathrm{NaCl}$ concentrations on the stability of AgNP@PVP is represented in Figure 4. According to the results, increasing $\mathrm{NaCl}$ content somewhat decreased the average hydrodynamic diameter of the samples and caused an increase in their zeta potential. The UV-Vis results indicate $\mathrm{AgCl}$ generation, but this was not accompanied by zeta potential decrease, which was previously observed during the $\mathrm{pH}-$ related measurements. No SPR blueshift was detected (in fact, redshifts were visible on the absorbance spectra). Therefore, the decreased Z-average values cannot be explained solely by $\mathrm{AgNP}$ degradation despite that $\mathrm{AgCl}$ formation was substantial on physiological $\mathrm{NaCl}$ concentrations according to the UV-Vis graphs.

Upon reviewing the related literature, the interconnection of PVP molecules through PVP-water-PVP hydrogen bonds seems a plausible reason for these observations [35]. As the increasing $\mathrm{NaCl}$ concentration led to a decreased Z-average value while simultaneously increased the zeta potential of the nanoparticles, it can be hypothesized that the increasing $\mathrm{Na}^{+}$ion presence at the slipping plane of the nanoparticles interfered with the interconnecting H-bonds among PVP chains, which resulted in a decrease of average aggregate size, illustrated in Scheme 1. This explains the observed optical changes within the samples since changes in the dielectric constant due to increasing electrolyte concentration can affect surface plasmon resonance [24]. 


\section{$\mathrm{NaCl}$ Dependence: DLS}

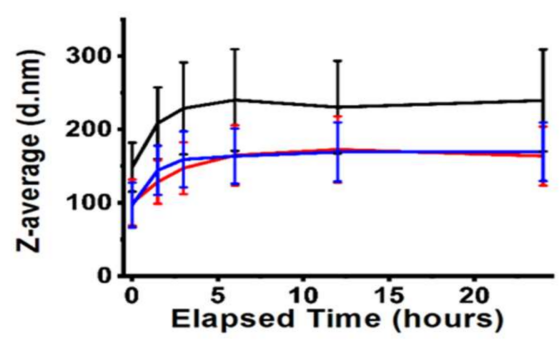

\section{UV-Vis}

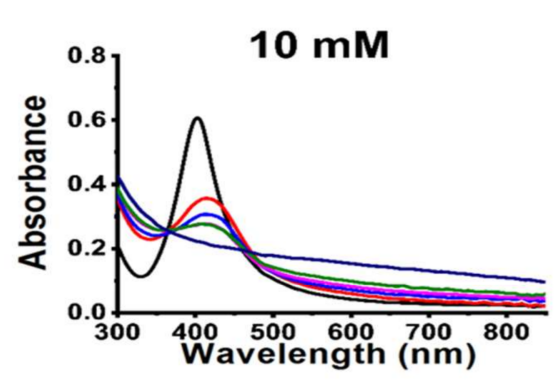

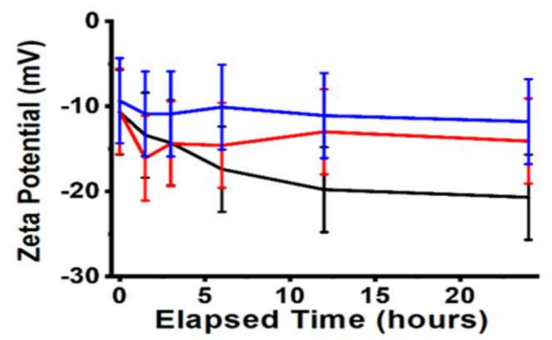

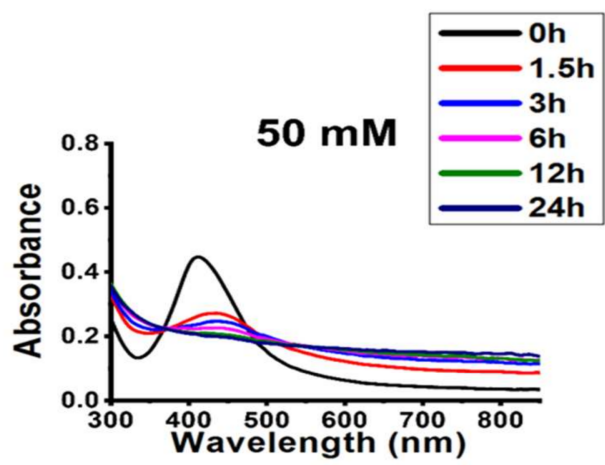

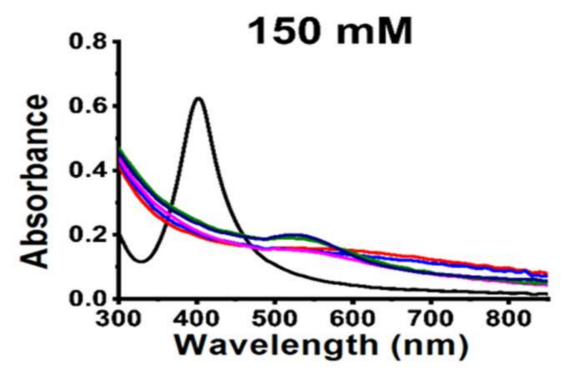

Figure 4. The effect of physiological $\mathrm{NaCl}$ concentrations on the stability of AgNP@PVP. Average hydrodynamic diameter (Z-average) and zeta potential changes (top panels) obtained by dynamic light scattering (DLS) and UV-Vis absorbance spectra (bottom panels) of polyvinyl-pyrrolidonestabilized silver nanoparticles, recorded under various $\mathrm{NaCl}$ concentrations over a 24-h timeframe.

\subsubsection{The Effect of Glucose and Glutamine}

According to Figures 5 and 6, the addition of small biomolecules to the colloids induced similar changes in the aggregation behavior of silver nanoparticles, regardless of the chemical composition or the concentration of the given biomolecule. Based on the DLS measurements taken at the early time points (between 1.5 and $6 \mathrm{~h}$ ), the presence of glucose in the physiological concentration and the application of glutamine in a concentration typically used in in vitro cell culture systems both decreased aggregate diameters and zeta potentials, ultimately converging towards the reference experiments (where the measurement was carried out on $\mathrm{pH} 7.2$ without interfering agents). The UV-Vis spectra of the colloids placed under these circumstances imply that the zeta potential results cannot be simply explained by $\mathrm{AgCl}$ formation as the characteristic AgNP SPR peaks were detectable (albeit redshifted) even after $24 \mathrm{~h}$. These results suggest that the investigated biomolecules get adsorbed on the NP surfaces, which feature moderately improved chemical and colloidal stability [36,37]. This surface adsorption (Scheme 1) is commonly referred to as biomolecular corona formation in more complex systems, and it can affect the optical and colloidal properties of AgNPs [25,38,39]. 
Glucose Dependence:

DLS

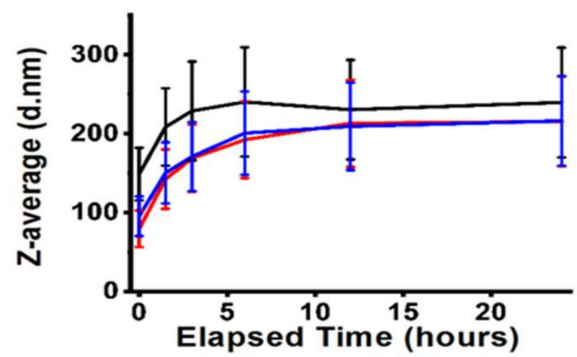

UV-Vis
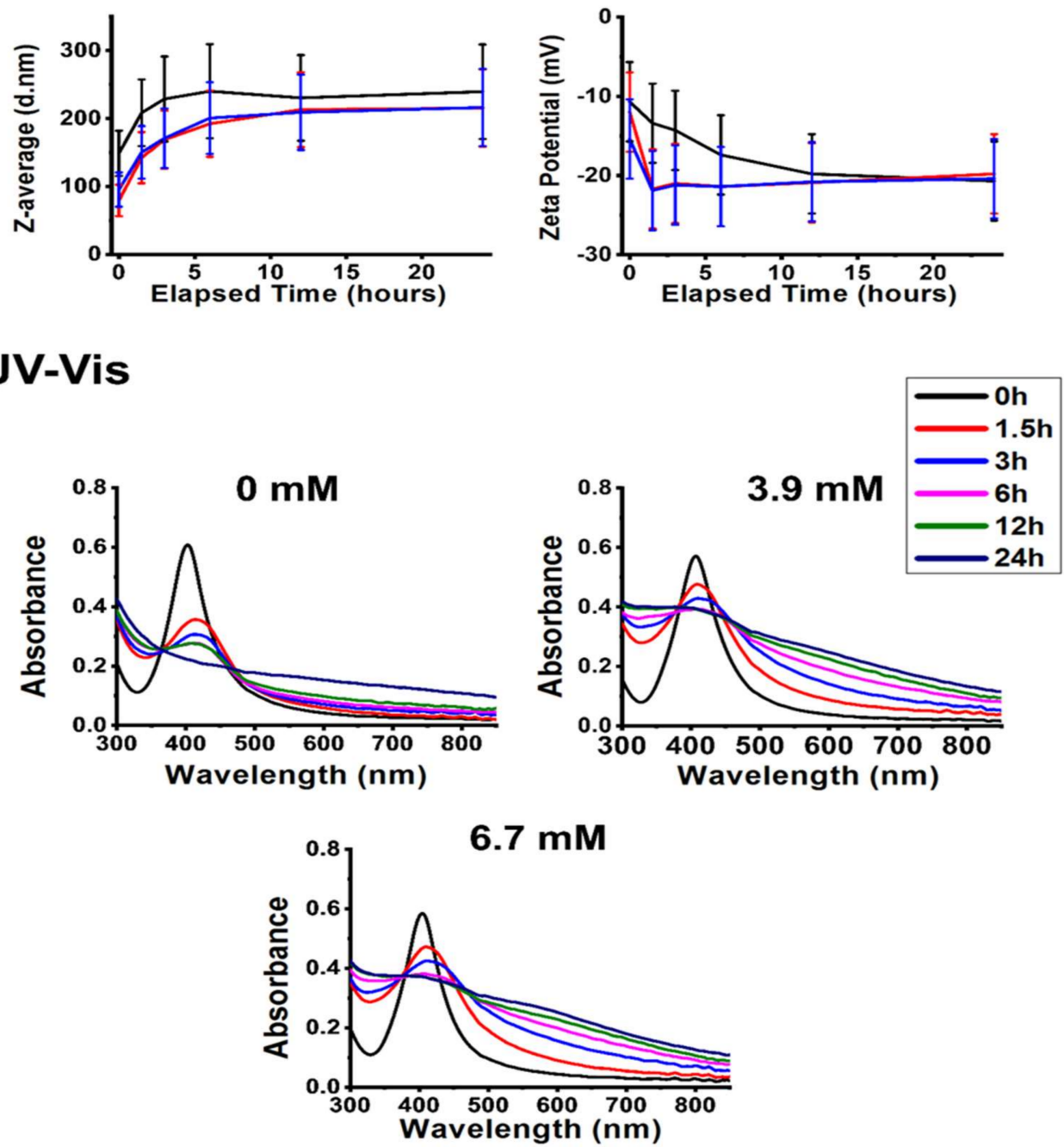

Figure 5. Dynamic light scattering (DLS) results (top), represented as average hydrodynamic diameter (Z-average) and zeta potential changes, and UV-Vis absorbance spectra (bottom) of polyvinylpyrrolidone-stabilized silver nanoparticles over $24 \mathrm{~h}$ under physiological glucose concentrations. 


\section{Glutamine Dependence: DLS}

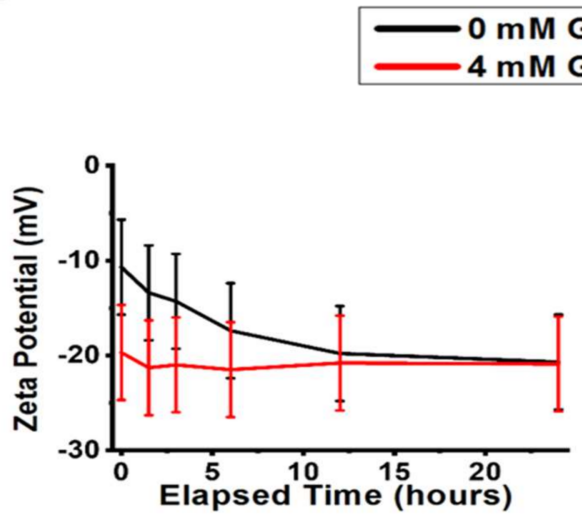

\section{UV-Vis}
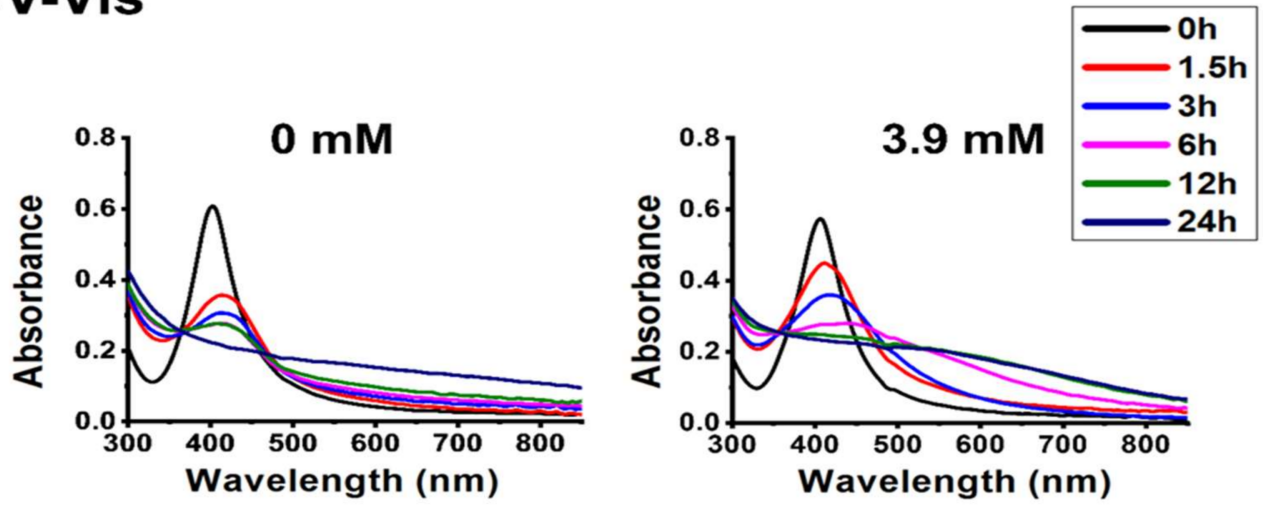

Figure 6. The presence of glutamine affects the stability of polyvinyl-pyrrolidone-stabilized silver nanoparticles. The average hydrodynamic diameter (Z-average) and zeta potential values were assessed by dynamic light scattering (DLS) (top) and UV-Vis absorbance spectra (bottom) of AgNP@PVP, recorded over $24 \mathrm{~h}$ in the presence of glutamine.

\subsubsection{The Effect of Cell Culture Medium Components}

In this section, the effect of two different cell culture components, Dulbecco's modified Eagle's medium (DMEM) and fetal bovine serum (FBS), was investigated on nanoparticle aggregation. From a chemical standpoint, DMEM can be considered a complex salt solution, and FBS a mixture of various biomolecules, overwhelmingly proteins [40]. Recognizing these solutions as chemically similar analogs for an environment of differing $\mathrm{NaCl}$ concentrations, tested previously, and another environment containing biomolecules such as glucose/glutamine, also examined in the preceding experiments, the data gathered here can be easily compared with the results described above in the manuscript.

Compared to the control experiment, the addition of DMEM slightly decreased the average hydrodynamic diameter and zeta potential and simultaneously stabilized the SPR absorbance of the particles. As a result of those experiments, where we assessed the effects of $\mathrm{NaCl}$ on particle aggregation, the accumulation of $\mathrm{Na}^{+}$ions around the slipping plane was hypothesized as the guiding feature behind the size decrease of AgNP@PVP aggregates. In those experiments, this phenomenon was accompanied by $\mathrm{AgCl}$ formation, indicated by $\zeta$-potential decrease and UV-Vis spectral changes. Here, in the presence of DMEM, we found a similar trend (Figure 7); however, silver chloride precipitation was not detected based on the UV-Vis spectra. The observation can be justified by inspecting the composition of DMEM. The same ionic strength is achieved by the addition of DMEM as $\mathrm{NaCl}$ (see in Section 2.3.2), only with a lower $\mathrm{Cl}^{-}$content; thus, the chemical stability of AgNPs was not so severely compromised under these conditions as within the milieu of the $\mathrm{NaCl}$ solution. 


\section{Medium Dependence: DLS}
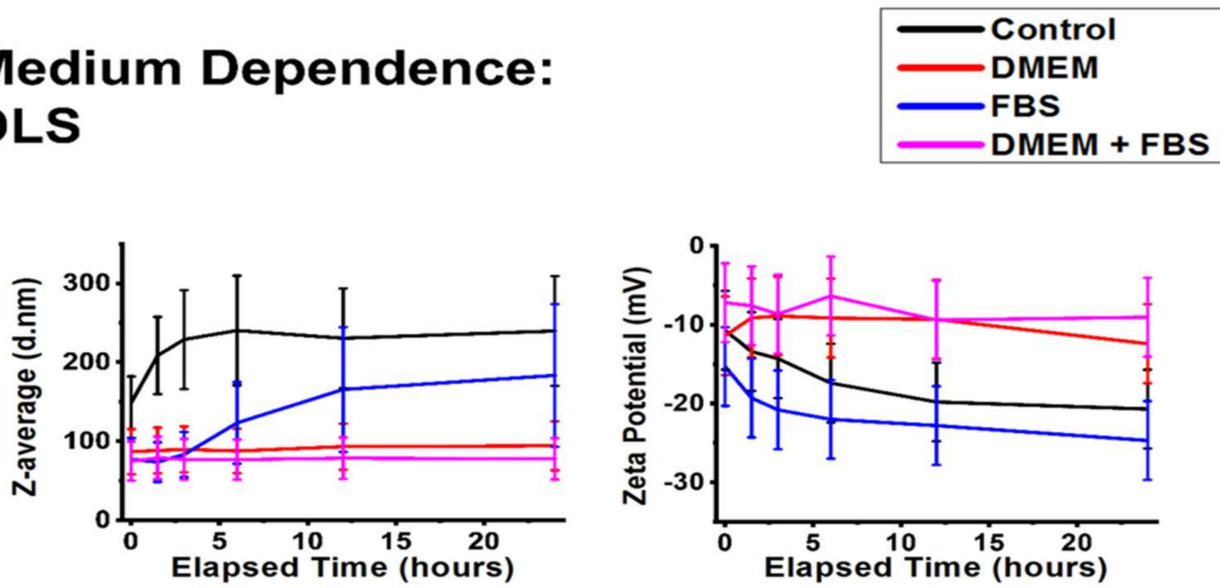

\section{UV-Vis}
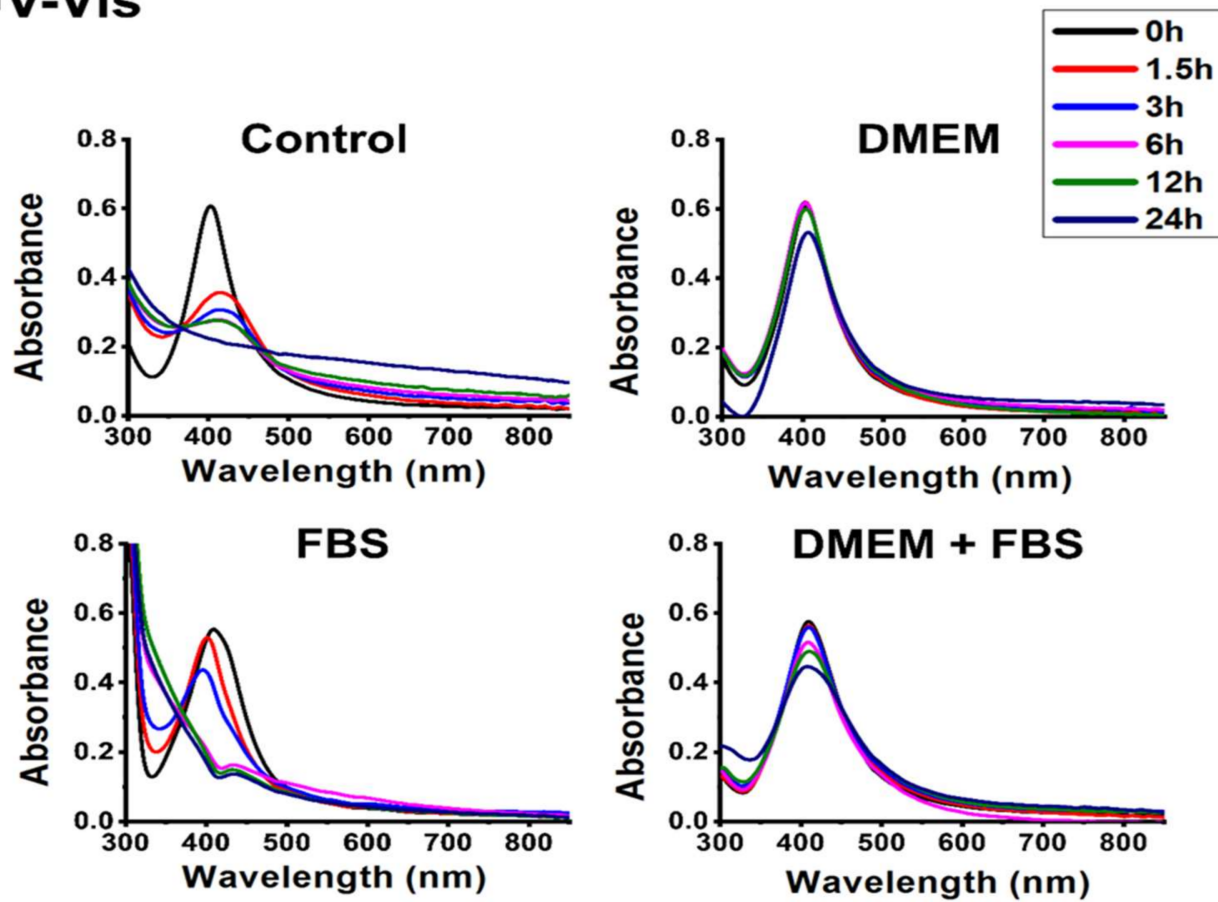

Figure 7. Aggregation behavior of silver nanoparticles characterized by dynamic light scattering (DLS) measurements (top) (average hydrodynamic diameter (Z-average) and zeta potential) and UV-Vis absorbance spectra (bottom) in the presence of cell culture components DMEM and FBS over a $24 \mathrm{~h}$ interval.

FBS induced analogous changes in the stability of AgNP@PVP just as glucose or glutamine did, with a moderately decreased Z-average and increased zeta potential. However, the presence of FBS induced a rather unique effect on the SPR of AgNPs, revealing interesting details of the mechanisms associated with nanosilver degradation due to $\mathrm{NaCl}$. According to the literature, the observed changes of SPR characteristics in the UV-Vis spectra can be attributed to the formation of clusters consisting of silver or silver chloride [41,42]. These clusters were most likely formed in the previous experiments as well but were immediately converted to bulk $\mathrm{AgCl}$ precipitation. The presence of FBS, however, prevented this process by creating a protective corona around the clusters. At the same time, it also displays how the chemical degradation of AgNPs due to $\mathrm{NaCl}$ involves a certain degree of particle fragmentation. These observations highlight that biomolecular coronas are not simply accessories for immune reactions, which is their most prominently 
discussed feature in the literature, but manifest an even more valuable characteristic as colloidal stabilizers of nanoparticles under biorelevant conditions [18,39,43,44].

As DMEM and FBS both improved the overall chemical and colloidal stability of AgNP@PVP particles separately, their combination was expected to exert a positive effect. Indeed, the DMEM+FBS experiment provided the smallest detected aggregate sizes within the entire work. The $\zeta$-potential of the system increased similarly to what was observed in the presence of only FBS, indicating considerable surface adsorption of various compounds. In contrast, the UV-Vis spectra of AgNP@PVP did not change substantially throughout the experiment despite a slight broadening, redshift, and intensity decline, which complemented the corona formation suggested by the zeta potential results.

The information gathered from the experiments revealed that during the biological application of AgNP@PVP, chemical stability rather than colloidal stability is the main point of contention; the latter proved to be the biggest weakness of nanosilver samples prepared with electrostatic stabilization, e.g., citrate groups [18].

\subsection{Biological Activity}

\subsubsection{Toxicity on Human Cells}

In order to evaluate how cytotoxic propensity can be influenced by the different aggregation states of AgNPs, we decided to use a two-step MTT cell viability assay-based approach, utilizing human cervical cancer (HeLa) cells as well as immortalized human keratinocyte $(\mathrm{HaCaT})$ cells.

For these analyses, in the first step, cell viability was measured after exposing both cell lines to AgNP@PVP in different concentrations to obtain the corresponding $\mathrm{IC}_{50}$ values. The $\mathrm{IC}_{50}$ values on HeLa cells were found to be $15.22 \mathrm{ppm}$, whereas IC $\mathrm{C}_{50}$ values on $\mathrm{HaCaT}$ cells were $2.74 \mathrm{ppm}$. Then, in the second step, the changes in AgNP cytotoxicity as the function of particle aggregation were explored in a time-dependent manner. For this, different silver nanoparticle aggregation states were formulated by adding $150 \mathrm{mM} \mathrm{NaCl}$ to silver samples for $0,1.5,3,6,12$, and $24 \mathrm{~h}$ prior to the beginning of the toxicity assays. We selected this approach based on our preliminary results and previous contributions, which indicated that among the tested experimental conditions, the electrolyte concentration had the greatest impact on nanoparticle stability [18,19]. AgNP samples of differing aggregation degrees (NaCl-treated AgNP samples) were created at nanoparticle concentrations corresponding to the respective cell line-specific $\mathrm{IC}_{50}$; then, the cells were incubated for $24 \mathrm{~h}$ with these AgNP samples, followed by MTT experiments. This way, the aggregation-dependent toxicity of AgNP@PVP samples could be measured.

The MTT viability assays performed on HeLa cervical cancer cells and the HaCaT keratinocyte cell line revealed that the toxicity of PVP-stabilized nanoparticles remained largely unchanged with increasing aggregation grades (Figure 8). These results implied that no aggregates were formed and all investigated AgNPs were stable and preserved their colloidal stability and toxicity for the entire aggregation timeframe. Despite the observed colloidal stability, by this point, we knew that the chemical stability of the particles was, in fact, compromised (Figures 2 and 4) since these particles were prone to decomposition upon surface contact with $\mathrm{Cl}^{-}$ions through $\mathrm{AgCl}$ formation. Therefore, the results reflected the combined effect of $\mathrm{AgNP} @ \mathrm{PVP}$ and the generated $\mathrm{AgCl}$. Consequently, the $\mathrm{IC}_{50}$ values of $\mathrm{AgCl}$ on both cell lines were determined as well, which were comparable with the $\mathrm{IC}_{50}$ values of AgNP@PVP ( $\mathrm{IC}_{50}$ values of AgCl: 10.37 ppm on HeLa cells; 2.31 ppm on HaCaT cells). These results are in good agreement with the fact that toxicity was maintained at the same level throughout the experimental time frame. 
HaCaT

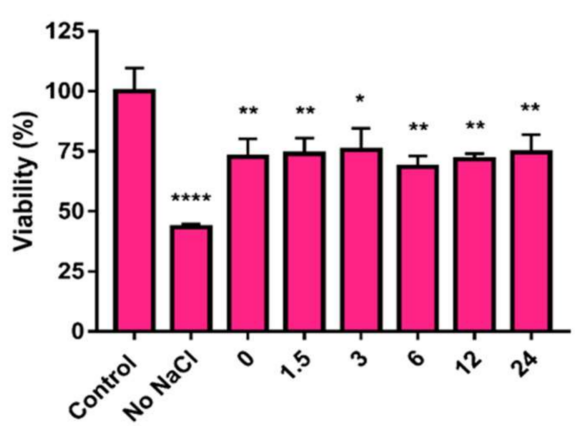

Aggregation time (hours)
HeLa

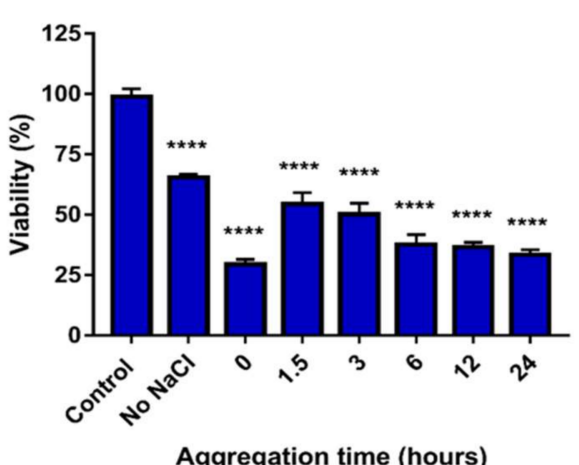

Figure 8. The effect of nanoparticle aggregation on the cytotoxicity exhibited on immortalized human keratinocyte $(\mathrm{HaCaT})$ cells and human cervical cancer (HeLa) cells. Increasing aggregation states of PVP-stabilized silver nanoparticles were created by incubating the particles with $150 \mathrm{mM} \mathrm{NaCl}$ for different time periods $(0,1.5,3,6,12$, and $24 \mathrm{~h})$ prior to cell treatments. The statistical significance of the experiments was calculated through unpaired $t$-tests and are marked with * $(p \leq 0.05)$, $* *(p \leq 0.01)$, and $* * * *(p \leq 0.0001)$.

\subsubsection{Antimicrobial Activity}

In order to provide the most reliable results, the changes in antibacterial and antifungal activities of AgNP@PVP samples in differing aggregation grades were also examined.

For these, a similar procedure to the cytotoxicity studies on human cells was used. The minimal inhibitory concentrations (MICs) were defined first and then used throughout the microbiology experiments. Then, the antimicrobial effect of the NaCl-treated silver samples (where the aggregation grade was modulated by the addition of $\mathrm{NaCl}$ ) was tested against Cryptococcus neoformans, Bacillus megaterium, and Escherichia coli strains. The selection of the pathogens was based on the idea of having a representative strain of Gram (+) and Gram (-) bacteria as well as of eukaryotic microorganisms.

The minimal inhibitory concentration of AgNP@PVP was 3.90 ppm for B. megaterium and E. coli and $1.95 \mathrm{ppm}$ for $C r$. neoformans. We found that the viability of all three strains remained unaffected; the antimicrobial efficiency of the nanoparticles remained the same even under growing nanoparticle aggregation grades (Figure 9). This was in accordance with the cytotoxicity results. In all cases, microbial cell viability was the lowest when cells were exposed to AgNPs that were not previously treated with $\mathrm{NaCl}$. In the case of samples where $\mathrm{NaCl}$ was supplemented to induce particle aggregation, the toxicity was not affected by the loss of particle stability; only a small difference in the extent of the toxic effect could be noticed. Cr. neoformans was the most sensitive, and E. coli was the most resistant strain against AgNP@PVP. In line with the human toxicity data, we concluded that AgNP@PVP samples could retain a significant degree of inhibitory activity on the tested microbes for the entire experimental timespan ( above $80 \%$ effectiveness for all strains). 
Cr. Neoformans

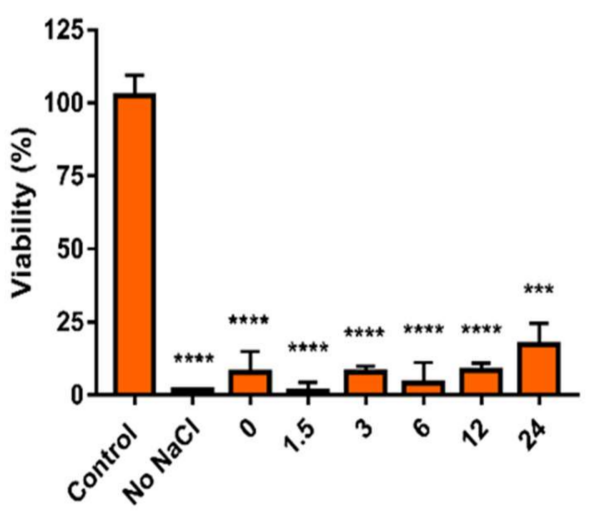

Aggregation time (hours)
B. megaterium

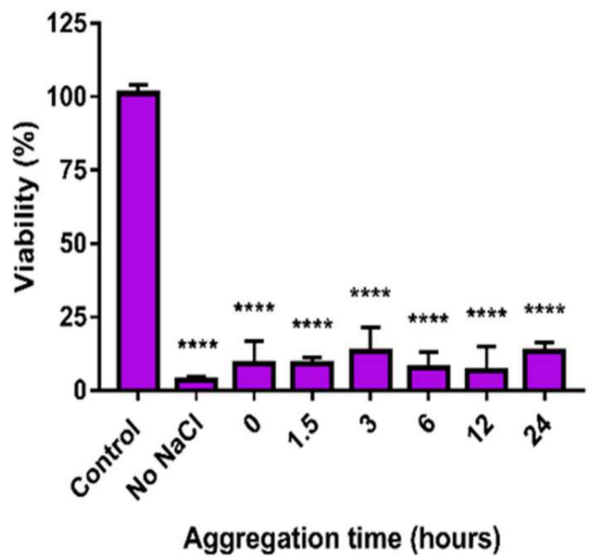

E.coli

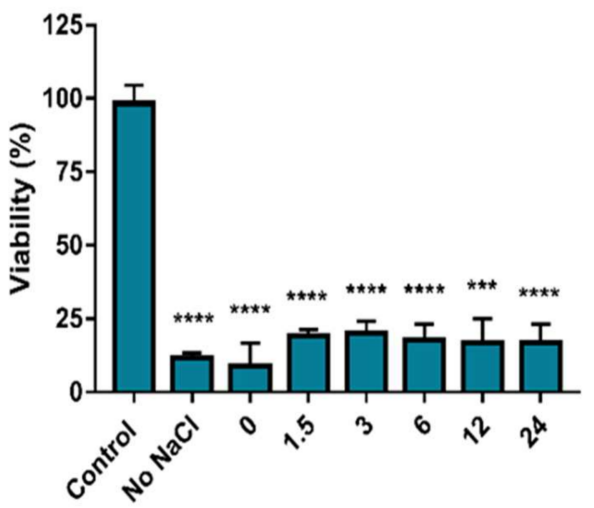

Aggregation time (hours)

Figure 9. The effect of nanoparticle aggregation on the antimicrobial activity against $\mathrm{Cr}$. neoformans, B. megaterium, and E. coli. Increasing aggregation degree was achieved by incubating the particles with $150 \mathrm{mM} \mathrm{NaCl}$ for different time periods $(0,1.5,3,6,12$, and $24 \mathrm{~h})$ prior to treatments. The statistical significance of the experiments was calculated through unpaired $t$-tests and are marked with $^{* * *}(p \leq 0.001)$, and ${ }^{* * * *}(p \leq 0.0001)$.

It is not sufficiently emphasized, but nanoparticle stability is an important physicochemical feature that must be seriously considered when nanoscale materials are designed and produced since aggregation could be a huge barrier in efficient nanoparticle application. It is known that the various materials used for stabilization have a defining role in determining and fine-tuning the biological activities of the obtained nanoparticles, such as toxicity, anticancer features, or antimicrobial activity, all of which can be seriously compromised by particle aggregation [45]. In our previous research, we found that the electrostatic repulsion provided by the most commonly used stabilization method, i.e., citrate capping, results in nanoparticles that are highly susceptible towards aggregation under biorelevant conditions, which can lead to the complete loss of their toxicity in specific circumstances. The results suggest that the loss of toxicity can be counteracted by either increasing the primary nanoparticle size or the introduction of additional repulsive forces among particles or through stronger (electrosteric) capping or biomolecular corona formation [18,19,46,47]. The use of PVP as a biocompatible and robust steric stabilizer was a suitable continuation of this research, as PVP already has numerous relevant uses, from the food industry to the pharmaceutical industry and drug delivery [32,33]. Our comprehensive chemical and biological data verify that PVP-stabilized silver nanoparticles are quite resistant against aggregation under biorelevant conditions and demonstrate the highest time-dependent 
toxicity we have reported to date. However, the chemical stability of the particles was weaker than what we observed within the systems of our previous contributions, where electrostatic repulsive interactions were also utilized. Interestingly, the degradation of the particles via $\mathrm{AgCl}$ precipitation did not substantially affect the toxicity of the particles, indicating that colloidal stability might be more important than chemical stability regarding the longevity of silver nanoparticle toxicity. However, it should be considered that biomedical (especially therapeutic) applications generally require well-defined, unchanging, long-term chemical characteristics and biological effects, which, according to our results, might not be plausible through PVP capping alone. On the other hand, certain environmental applications (for instance, wastewater treatment) could potentially benefit from a nanoparticulate system that can be administered in a way where the initial nano-size properties can be effectively utilized while the separation of the nanomaterial can be realized through the removal of the water-insoluble precipitation that is generated over time.

\section{Materials and Methods}

\subsection{Nanoparticle Synthesis}

All chemicals were purchased from Merck KGaA (Darmstadt, Germany). PVPstabilized silver nanoparticles (AgNP@PVP) were prepared by chemical reduction based on the approach proposed by Wan et al. with slight adjustments, the same procedure that we used in our previous contributions $[18,48,49]$. While the original method is based on the chemical reduction of silver nitrate $\left(\mathrm{AgNO}_{3}\right)$ by sodium borohydride $\left(\mathrm{NaBH}_{4}\right)$ with sodium citrate capping, here we used PVP in two different (40k and 55k) average molecular weights as stabilizing agents. Firstly, $0.17 \mathrm{~g}$ PVP (40k or 50k) was dispersed in $96 \mathrm{~mL}$ of ion-exchanged water and was heated to $70{ }^{\circ} \mathrm{C}$ in a beaker. Then, $2 \mathrm{~mL} 1 \% w / v \mathrm{AgNO}_{3}$ solution was administered to the polymer solution, followed by the dropwise addition of $2 \mathrm{~mL} 0.1 \% w / v$ freshly prepared sodium borohydride. The resulting AgNP samples were kept at $70{ }^{\circ} \mathrm{C}$ under vigorous stirring for $1 \mathrm{~h}$ and, afterward, left to cool to room temperature. Any remaining contaminants were removed by dialysis using a cellulose membrane. Ultimately, the volumes of the samples were set to $85 \mathrm{~mL}$; thus, each suspension was $150 \mathrm{ppm}$ regarding AgNPs and $2 \mathrm{mg} / \mathrm{mL}$ (or $13.3 \mathrm{mg} / \mathrm{mg}$ AgNP) for PVP, respectively.

\subsection{Nanoparticle Characterization}

The morphological characteristics of the nanoparticles were assessed by transmission electron microscopy (TEM) with an FEI Tecnai $\mathrm{G}^{2} 20$ X-Twin instrument using $200 \mathrm{kV}$ accelerating voltage (FEI Corporate Headquarters, Hillsboro, OR, USA). The size distribution of the samples was demonstrated through histograms assessed by evaluating 15 representative images. The crystallinity and, thus, the chemical composition of the particles was verified through electron diffraction (ED).

\subsection{Selection of the Proper AgNP@PVP System Based on Chemical Stability}

Preliminary experiments were performed on an Ocean Optics 355 DH-2000-BAL UV-Vis spectrophotometer (Halma PLC, Largo, FL, USA) to investigate the chemical stability of AgNPs in the abundance of chloride ions by observing changes in the surface plasmon resonance (SPR) of the nanosilver samples [50]. To identify the most chemically resistant AgNP@PVP system produced via the synthesis method, both AgNP samples (stabilized by $40 \mathrm{k}$ and $55 \mathrm{k}$ molecular weight PVP, respectively) were examined by UV-Vis spectroscopy. The PVP concentrations of the as-prepared samples were adjusted with the appropriate molecular weight polymer to $2,4,8$, and $10 \mathrm{mg} / \mathrm{mL}$ (or 13.3, 26.6, 53.3, and $66.6 \mathrm{mg} / \mathrm{mg}$ AgNP, respectively), and their initial SPR spectra were collected after a $30 \mathrm{~s}$ long sonication. Then, the samples' $\mathrm{NaCl}$ concentrations were set to $100 \mathrm{mM}$, and their UV-Vis absorbance was once again measured after $20 \mathrm{~h}$. The best sample regarding the molecular weight and concentration of PVP was identified and used for the subsequent colloidal stability experiments. 


\subsection{Aggregation Behavior Assays}

We have previously demonstrated that biologically relevant conditions may affect the stability and the behavior of citrate-capped and green-tea-extract-stabilized silver nanoparticles; furthermore, in a following contribution, we highlighted the importance of primer particle size as a parameter with profound importance on colloidal stability and, hence, toxicity using a novel experimental setup of our own $[18,19]$. As the applied methodological approach proved to be adequate and highly competent to reliably characterize these features, we performed the same methodology here to describe the aggregation propensity of the selected AgNP@PVP sample. The effect of $\mathrm{pH}$, sodium chloride, glucose, and glutamine, as well as Dulbecco's modified Eagle medium (DMEM, Sigma-Aldrich, Saint Louis, MO, USA) and fetal bovine serum (FBS, Sigma-Aldrich, Saint Louis, MO, USA) (both in water and DMEM) were investigated at biologically and environmentally important values and concentrations, according to Table 1 [51-55]. Each experiment lasted $24 \mathrm{~h}$, with measurements performed at the $0,1.5,3,6,12$, and $24 \mathrm{~h}$ checkpoints. At the checkpoints, the characteristic UV-Vis spectra of the samples were captured; furthermore, the Z-average (using dynamic light scattering) and $\zeta$-potential of the samples were also investigated on a Malvern Zetasizer Nano ZS instrument (Malvern Instruments, Malvern, UK). In all experiments, the concentration of AgNPs was set to $22 \mathrm{ppm}$. This nanoparticle concentration was selected because this concentration corresponded to $\sim 0.8$ absorbance, allowing the low-noise and sensitive detection of spectral changes; furthermore, this way, all considered biorelevant agents were in concentrations of higher orders of magnitude compared to that of nanoparticles, adequately simulating the lifelike circumstances. Unless otherwise stated, all measurements were performed at $\mathrm{pH} 7.2$ and $37^{\circ} \mathrm{C}$, with a $10 \mathrm{mM} \mathrm{NaCl}$ background salt concentration to negate long-distance particle interactions; furthermore, the $\mathrm{pH}$ of the samples was always checked before measurements and corrected when necessary [56].

Table 1. List of applied biorelevant conditions; $\left({ }^{\dagger}\right)$ represents the reference point for the measurements. All samples were measured at $37^{\circ} \mathrm{C}, \mathrm{pH} 7.2$, with a $10 \mathrm{mM} \mathrm{NaCl}$ electrolyte background unless otherwise stated.

\begin{tabular}{cc}
\hline Condition & Value(s) \\
\hline $\mathrm{pH}$ & $3 ; 5 ; 7.2^{\dagger} ; 9$ \\
$\mathrm{NaCl}$ concentration $(\mathrm{mM})$ & $10 ; 50 ; 150$ \\
Glucose concentration $(\mathrm{mM})$ & $3.9 ; 6.7$ \\
Glutamine concentration $(\mathrm{mM})$ & 4 \\
DMEM $(v / v \%)$ & 45 \\
FBS $(v / v \%$, both in water and DMEM mixture) & 5 \\
\hline
\end{tabular}

\subsection{Cytotoxicity Assays}

Human cervical carcinoma (HeLa) cells and immortalized human keratinocyte (HaCaT) cells were purchased from American Type Culture Collection (ATCC, Manassas, VA, USA). Cells were routinely maintained in DMEM containing $4.5 \mathrm{~g} / \mathrm{L}$ glucose (SigmaAldrich, Saint Louis, MO, USA), supplemented with 10\% FBS, 2 mM L-glutamine, $0.01 \%$ streptomycin, and $0.005 \%$ penicillin (all components were obtained from Sigma-Aldrich, Saint Louis, MO, USA). Cells were cultured under standard conditions in a $37^{\circ} \mathrm{C}$ incubator at $5 \% \mathrm{CO}_{2}$ in $95 \%$ humidity.

Similar to our previous experiments, a well-defined system was employed to measure the changes in AgNP cytotoxicity affected by particle aggregation in a time-dependent manner, as proposed by our previous contributions [18,19]. A two-step approach based on MTT assays was carried out, where, first, the $\mathrm{IC}_{50}$ values of the PVP-stabilized silver sol were determined as a reference for the toxicity of non-aggregated particles for both the cancerous and healthy human cell lines, and the effect of aggregation was demonstrated on these concentrations in a subsequent round of MTT assays. For this, both types of cells were seeded into 96-well plates in a $10^{4}$ cells/well density and treated on the following day with silver nanoparticles in increasing concentrations. After $24 \mathrm{~h}$ treatments, cells 
were washed with PBS and incubated with a culture medium containing $0.5 \mathrm{mg} / \mathrm{mL}$ MTT reagent (Sigma-Aldrich, Saint Louis, MO, USA) for $1 \mathrm{~h}$ at $37^{\circ} \mathrm{C}$. The resulting formazan crystals were dissolved in DMSO (Sigma-Aldrich, Saint Louis, MO, USA), and the absorbance of the samples was measured at $570 \mathrm{~nm}$ using a SPECTROstar Nano plate reader (BioTech-Hungary, Budapest, Hungary). Absorption values of the untreated control samples were considered $100 \%$ viability. The experiments were carried out three times using four independent biological replicates. $\mathrm{IC}_{50}$ was calculated using the dose-response curve based on the viability results.

Thereafter, different silver nanoparticle aggregation states were formulated by incubating the particles (at the $\mathrm{IC}_{50}$ concentration) with $150 \mathrm{mM} \mathrm{NaCl}$ for different time periods $(0,1.5,3,6,12$, and $24 \mathrm{~h})$ prior to cell treatments. Subsequently, the MTT assay was performed, as described above, to explore the changes in cell viability caused by particle aggregation.

\subsection{Assessment of the Antimicrobial Activity}

To evaluate the changes in antimicrobial activities caused by particle aggregation, first, a microdilution method was employed to determine the minimum inhibitory concentration (MIC) of the nanosilver sample as a reference for the toxicity of non-aggregated particles. The MIC was defined against microbial strains that were the subjects of our previous studies [18,19]. The initial cell concentration of Cryptococcus neoformans IFM 5844 (IFM; Research Center for Pathogenic Fungi and Microbial Toxicoses, Chiba University), Bacillus megaterium SZMC 6031, and Escherichia coli SZMC 0582 (SZMC: Szeged Microbiology Collection) strains in RPMI 1640 medium (Sigma-Aldrich, Saint Louis, MO, USA) was $10^{5}$ cells $/ \mathrm{mL}$, respectively. Two-fold dilution series of nanoparticles were made in the $0-75 \mathrm{ppm}$ concentration range. From each cell suspension, $50 \mu \mathrm{L}$ aliquots were loaded into the wells of a 96-well microplate, and $50 \mu \mathrm{L}$ of individual AgNP dilutions were added to the cells. Suspensions supplemented with $50 \mu \mathrm{L}$ RPMI 1640 were used as growth control. Then, microplates were incubated at $30^{\circ} \mathrm{C}$ for $48 \mathrm{~h}$, and the optical density of the cultures was detected at $620 \mathrm{~nm}$ using a SPECTROstar Nano plate reader (BMG LabTech, Offenburg, Germany). The experiments were carried out three times in triplicates.

The antimicrobial activity of the aggregated nanoparticles was tested on the abovementioned strains with the same approach described in the previous paper [18]. Briefly, suspensions of $10^{5}$ cells $/ \mathrm{mL}$ were prepared in RPMI 1640 medium. Then, $50 \mu \mathrm{L}$ aliquots from Cr. neoformans, B. megaterium, and E. coli cell suspensions were loaded into the wells of a 96-well microplate, and, this time, $50 \mu \mathrm{L}$ of the individual aggregated nanoparticle samples were added to the cells. The different silver nanoparticle aggregation states were created by incubating the particles (at the MIC concentration) with $150 \mathrm{mM} \mathrm{NaCl}$ for different time periods $(0,1.5,3,6,12$, and $24 \mathrm{~h})$ before cell treatments. Suspensions supplemented with $50 \mu \mathrm{L}$ RPMI 1640 were used as growth control, while suspensions with non-aggregated nanoparticles were used as controls of toxicity. The light absorbance of the samples was measured at $620 \mathrm{~nm}$ using a SPECTROstar Nano plate reader (BMG LabTech, Offenburg, Germany). The experiments were carried out three times in four replicates.

\subsection{Statistical Analysis}

All the experiments were carried out three times in four replicates. The final results of the cytotoxicity and antimicrobial assays were assessed using GraphPad Prism 7 (GraphPad Software, San Diego, CA, USA); the statistical significance of the experiments was calculated through unpaired $t$-tests, and their levels are marked as ${ }^{*}(p \leq 0.05),{ }^{* *}(p \leq 0.01)$, $* * *(p \leq 0.001)$, and ${ }^{* * * *}(p \leq 0.0001)$.

\section{Conclusions}

Silver nanoparticles are among the most biologically significant nano-sized materials due to their exceptional antimicrobial, anti-inflammatory, anticancer, and antiangiogenic properties. However, due to nanoparticle aggregation-a phenomenon often occurring 
in biological conditions-these advantageous characteristics are easily perturbed. In this work, the effectiveness of PVP as a silver nanoparticle-capping agent was assessed against biologically relevant materials and conditions. The first objective of our research was the synthesis and characterization of polymer-capped silver nanoparticle samples stabilized by 40k or 55k MW PVP, aiming to find a sample with high chemical uniformity and stability. In the following steps, through the subsequent aggregation behavior experiments, the sample possessing the best characteristics (AgNP@PVP, stabilized by 40k MW PVP in $13.3 \mathrm{mg} / \mathrm{mg}$ AgNP relative concentration) demonstrated robust colloidal stability against most of the investigated conditions apart from mildly acidic $\mathrm{pH}$, where the crosslinking of polymer chains became possible through $\mathrm{H}^{+}$bridges. Despite the outstanding colloidal stability of the sample, the chemical degradation of AgNPs through $\mathrm{AgCl}$ precipitation was detected in the presence of elevated $\mathrm{Cl}^{-}$concentrations. While chemical degradation could be observed with as low a $\mathrm{NaCl}$ concentration as $10 \mathrm{mM}$, biomolecular corona formation demonstrated a protective effect against precipitation via the surface adsorption of biomolecules on nanoparticle surfaces. In fact, corona formation revealed the presence of silver clusters in the precipitating samples, indicating that the chemical degradation of AgNPs under biorelevant conditions might involve nanoparticle fragmentation. Throughout the in vitro studies, AgNP@PVP demonstrated excellent and long-lasting toxicity against cancerous and non-cancerous human cell lines and microbial strains alike. In contrast with our previous contributions, it can be assessed that colloidal stability is more important than chemical stability for the longevity of silver nanoparticle toxicity, although when facing real biomedical challenges, such as in vivo therapeutic applications, extra attention must be paid to exclude the possibility of adverse reactions due to precipitation.

Author Contributions: Conceptualization, A.R., P.B., Z.K. and M.K.; methodology, A.R., P.B., N.I., E.B. and I.P.; software, A.R., P.B., N.I. and B.S.; validation, P.B. and A.R.; investigation, A.R., P.B., N.I., E.B., D.Z. and B.S.; resources, Z.K., I.P. and M.K.; data curation, N.I., B.S., P.B. and A.R.; writingoriginal draft preparation, A.R., P.B., M.K., N.I., I.P. and B.S.; writing-review and editing, M.K., A.R. and P.B.; visualization, N.I., B.S., A.R. and P.B.; supervision, M.K., Z.K. and I.P.; funding acquisition, Z.K., M.K. and I.P. All authors have read and agreed to the published version of the manuscript.

Funding: This research was supported by the New National Excellence Program of the Ministry for Innovation and Technology from the National Research, Development, and Innovation Fund (ÚNKP20-4-SZTE-580 for P.B. and ÚNKP-20-5-SZTE-655 for M.K.) and the János Bolyai Research Scholarship of the Hungarian Academy of Sciences (BO/00878/19/8 for M.K. and BO/00384/21/7 for A.R.). Financial support by the National Research, Development, and Innovation Office-NKFIH through projects GINOP-2.3.2-15-2016-00038 and GINOP-2.3.2-15-2016-00035 is gratefully acknowledged. The APC was funded by the Open Access publication funding of the University of Szeged.

Institutional Review Board Statement: Not applicable.

Informed Consent Statement: Not applicable.

Data Availability Statement: The data presented in this study are available on request from the corresponding author.

Conflicts of Interest: The authors declare no conflict of interest.

\section{References}

1. Rónavári, A.; Igaz, N.; Adamecz, D.; Szerencsés, B.; Molnar, C.; Kónya, Z.; Pfeiffer, I.; Kiricsi, M. Green Silver and Gold Nanoparticles: Biological Synthesis Approaches and Potentials for Biomedical Applications. Molecules 2021, 26, 844. [CrossRef] [PubMed]

2. Lee, S.H.; Jun, B.-H. Silver Nanoparticles: Synthesis and Application for Nanomedicine. Int. J. Mol. Sci. 2019, 20, 865. [CrossRef] [PubMed]

3. Xu, L.; Wang, Y.-Y.; Huang, J.; Chen, C.-Y.; Wang, Z.-X.; Xie, H. Silver nanoparticles: Synthesis, medical applications and biosafety. Theranostics 2020, 10, 8996-9031. [CrossRef] [PubMed]

4. Verma, P.; Maheshwari, S.K. Applications of Silver nanoparticles in diverse sectors. Int. J. Nano Dimens. 2019, $10,18-36$. 
5. Ivask, A.; Kurvet, I.; Kasemets, K.; Blinova, I.; Aruoja, V.; Suppi, S.; Vija, H.; Kakinen, A.; Titma, T.; Heinlaan, M.; et al. SizeDependent Toxicity of Silver Nanoparticles to Bacteria, Yeast, Algae, Crustaceans and Mammalian Cells In Vitro. PLoS ONE 2014, 9, e102108. [CrossRef] [PubMed]

6. Xu, R.; Wang, D.; Zhang, J.; Li, Y. Shape-Dependent Catalytic Activity of Silver Nanoparticles for the Oxidation of Styrene. Chem.-Asian J. 2006, 1, 888-893. [CrossRef] [PubMed]

7. Osonga, F.J.; Akgul, A.; Yazgan, I.; Akgul, A.; Eshun, G.B.; Sakhaee, L.; Sadik, O.A. Size and Shape-Dependent Antimicrobial Activities of Silver and Gold Nanoparticles: A Model Study as Potential Fungicides. Molecules 2020, 25, 2682. [CrossRef]

8. Rónavári, A.; Kovács, D.; Igaz, N.; Vágvölgyi, C.; Boros, I.M.; Konya, Z.; Pfeiffer, I.; Kiricsi, M. Biological activity of greensynthesized silver nanoparticles depends on the applied natural extracts: A comprehensive study. Int. J. Nanomed. 2017, 12, 871-883. [CrossRef]

9. Rónavári, A.; Igaz, N.; Gopisetty, M.K.; Szerencsés, B.; Kovács, D.; Papp, C.G.; Vágvölgyi, C.; Boros, I.M.; Kónya, Z.; Kiricsi, M.; et al. Biosynthesized silver and gold nanoparticles are potent antimycotics against opportunistic pathogenic yeasts and dermatophytes. Int. J. Nanomed. 2018, 13, 695-703. [CrossRef]

10. Landage, S.M.; Wasif, A.I.; Dhuppe, P. Synthesis of nanosilver using chemical reduction methods-Indian Journals. Int. J. Adv. Res. Eng. Appl. Sci. 2014, 3, 14-22.

11. El Badawy, A.M.; Luxton, T.P.; Silva, R.G.; Scheckel, K.; Suidan, M.T.; Tolaymat, T.M. Impact of Environmental Conditions (pH, Ionic Strength, and Electrolyte Type) on the Surface Charge and Aggregation of Silver Nanoparticles Suspensions. Environ. Sci. Technol. 2010, 44, 1260-1266. [CrossRef] [PubMed]

12. Jeevanandam, J.; Barhoum, A.; Chan, Y.S.; Dufresne, A.; Danquah, M.K. Review on nanoparticles and nanostructured materials: History, sources, toxicity and regulations. Beilstein J. Nanotechnol. 2018, 9, 1050-1074. [CrossRef] [PubMed]

13. Ajitha, B.; Reddy, Y.A.K.; Reddy, P.S.; Jeon, H.-J.; Ahn, C.W. Role of capping agents in controlling silver nanoparticles size, antibacterial activity and potential application as optical hydrogen peroxide sensor. RSC Adv. 2016, 6, 36171-36179. [CrossRef]

14. Prathna, T.; Chandrasekaran, N.; Mukherjee, A. Studies on aggregation behaviour of silver nanoparticles in aqueous matrices: Effect of surface functionalization and matrix composition. Colloids Surf. A Physicochem. Eng. Asp. 2011, 390, 216-224. [CrossRef]

15. Tejamaya, M.; Römer, I.; Merrifield, R.C.; Lead, J.R. Stability of Citrate, PVP, and PEG Coated Silver Nanoparticles in Ecotoxicology Media. Environ. Sci. Technol. 2012, 46, 7011-7017. [CrossRef] [PubMed]

16. Restrepo, C.V.; Villa, C.C. Synthesis of silver nanoparticles, influence of capping agents, and dependence on size and shape: A review. Environ. Nanotechnol. Monit. Manag. 2021, 15, 100428. [CrossRef]

17. El Badawy, A.M.; Scheckel, K.; Suidan, M.; Tolaymat, T. The impact of stabilization mechanism on the aggregation kinetics of silver nanoparticles. Sci. Total. Environ. 2012, 429, 325-331. [CrossRef]

18. Bélteky, P.; Rónavári, A.; Igaz, N.; Szerencsés, B.; Tóth, I.Y.; Pfeiffer, I.; Kiricsi, M.; Kónya, Z. Silver nanoparticles: Aggregation behavior in biorelevant conditions and its impact on biological activity. Int. J. Nanomed. 2019, 14, 667-687. [CrossRef]

19. Bélteky, P.; Rónavári, A.; Zakupszky, D.; Boka, E.; Igaz, N.; Szerencsés, B.; Pfeiffer, I.; Vágvölgyi, C.; Kiricsi, M.; Kónya, Z. Are Smaller Nanoparticles Always Better? Understanding the Biological Effect of Size-Dependent Silver Nanoparticle Aggregation Under Biorelevant Conditions. Int. J. Nanomed. 2021, 16, 3021-3040. [CrossRef]

20. Madkour, M.; Bumajdad, A.; Al-Sagheer, F. To what extent do polymeric stabilizers affect nanoparticles characteristics? Adv. Colloid Interface Sci. 2019, 270, 38-53. [CrossRef]

21. Dhand, V.; Soumya, L.; Bharadwaj, S.; Chakra, S.; Bhatt, D.; Sreedhar, B. Green synthesis of silver nanoparticles using Coffea arabica seed extract and its antibacterial activity. Mater. Sci. Eng. C 2016, 58, 36-43. [CrossRef] [PubMed]

22. Mehtab, S.; Zaidi, M.; Siddiqi, T.I. Designing Fructose Stabilized Silver Nanoparticles for Mercury(II) Detection and Potential Antibacterial Agents. Mater. Sci. Res. India 2018, 15, 241-249. [CrossRef]

23. Banua, J.; Han, J.I. Biogenesis of Prism-Like Silver Oxide Nanoparticles Using Nappa Cabbage Extract and Their p-Nitrophenol Sensing Activity. Molecules 2020, 25, 2298. [CrossRef] [PubMed]

24. García, M.A. Surface Plasmons in metallic nanoparticles: Fundamentals and applications. J. Phys. D Appl. Phys. 2012, $44,283001$. [CrossRef]

25. Alarcon, E.I.; Bueno-Alejo, C.; Noel, C.; Stamplecoskie, K.G.; Pacioni, N.; Poblete, H.; Scaiano, J.C. Human serum albumin as protecting agent of silver nanoparticles: Role of the protein conformation and amine groups in the nanoparticle stabilization. J. Nanoparticle Res. 2013, 15, 1-14. [CrossRef]

26. Badi'Ah, H.I.; Seedeh, F.; Supriyanto, G.; Zaidan, A.H. Synthesis of Silver Nanoparticles and the Development in Analysis Method. IOP Conf. Ser. Earth Environ. Sci. 2019, 217, 012005. [CrossRef]

27. Sharma, V.; Verma, D.; Okram, G.S. Influence of surfactant, particle size and dispersion medium on surface plasmon resonance of silver nanoparticles. J. Phys. Condens. Matter 2019, 32, 145302. [CrossRef]

28. Kang, H.; Buchman, J.T.; Rodriguez, R.S.; Ring, H.L.; He, J.; Bantz, K.C.; Haynes, C.L. Stabilization of Silver and Gold Nanoparticles: Preservation and Improvement of Plasmonic Functionalities. Chem. Rev. 2018, 119, 664-699. [CrossRef] [PubMed]

29. Yaqoob, A.A.; Umar, K.; Ibrahim, M.N.M. Silver nanoparticles: Various methods of synthesis, size affecting factors and their potential applications-A review. Appl. Nanosci. 2020, 10, 1369-1378. [CrossRef]

30. Fernando, I.; Zhou, Y. Impact of $\mathrm{pH}$ on the stability, dissolution and aggregation kinetics of silver nanoparticles. Chemosphere 2018, 216, 297-305. [CrossRef] 
31. Ezra, L.; O’Dell, Z.J.; Hui, J.; Riley, K.R. Emerging investigator series: Quantifying silver nanoparticle aggregation kinetics in real-time using particle impact voltammetry coupled with UV-vis spectroscopy. Environ. Sci. Nano 2020, 7, 2509-2521. [CrossRef]

32. Nair, B. Final Report On the Safety Assessment of Polyvinylpyrrolidone (PVP). Int. J. Toxicol. 1998, 17, 95-130. [CrossRef]

33. Franco, P.; De Marco, I. The Use of Poly(N-vinyl pyrrolidone) in the Delivery of Drugs: A Review. Polymers 2020, $12,1114$. [CrossRef] [PubMed]

34. Li, X.; Lenhart, J.J.; Walker, H.W. Dissolution-Accompanied Aggregation Kinetics of Silver Nanoparticles. Langmuir 2010, 26, 16690-16698. [CrossRef] [PubMed]

35. Brahma, S.; Rao, K.J.; Shivashankar, S. Rapid growth of nanotubes and nanorods of würtzite ZnO through microwave-irradiation of a metalorganic complex of zinc and a surfactant in solution. Bull. Mater. Sci. 2010, 33, 89-95. [CrossRef]

36. Costa, C.; Medronho, B.; Filipe, A.; Mira, I.; Lindman, B.; Edlund, H.; Norgren, M. Emulsion Formation and Stabilization by Biomolecules: The Leading Role of Cellulose. Polymers 2019, 11, 1570. [CrossRef]

37. Shu, M.; He, F.; Li, Z.; Zhu, X.; Ma, Y.; Zhou, Z.; Yang, Z.; Gao, F.; Zeng, M. Biosynthesis and Antibacterial Activity of Silver Nanoparticles Using Yeast Extract as Reducing and Capping Agents. Nanoscale Res. Lett. 2020, 15, 14. [CrossRef]

38. Riviere, J.E.; Scoglio, C.; Sahneh, F.D.; Monteiro-Riviere, N.A. Computational approaches and metrics required for formulating biologically realistic nanomaterial pharmacokinetic models. Comput. Sci. Discov. 2013, 6, 014005. [CrossRef]

39. Hadjidemetriou, M.; Kostarelos, K. Evolution of the nanoparticle corona. Nat. Nanotechnol. 2017, 12, 288-290. [CrossRef]

40. Sigma-Aldrich Dulbecco's Modified Eagle's Medium Product Information. Available online: https://www.sigmaaldrich.com/ content/dam/sigma-aldrich/docs/Sigma/Formulation/d5796for.pdf (accessed on 9 September 2020).

41. Pal, N.K.; Kryschi, C. A facile synthesis of highly stable and luminescent Ag clusters: A steady-state and time-resolved spectroscopy study. Phys. Chem. Chem. Phys. 2015, 17, 1957-1965. [CrossRef]

42. Trinh, N.D.; Nguyen, T.T.B.; Nguyen, T.H. Preparation and characterization of silver chloride nanoparticles as an antibacterial agent. Adv. Nat. Sci. Nanosci. Nanotechnol. 2015, 6, 45011. [CrossRef]

43. Pulido-Reyes, G.; Leganes, F.; Fernández-Piñas, F.; Rosal, R. Bio-nano interface and environment: A critical review. Environ. Toxicol. Chem. 2017, 36, 3181-3193. [CrossRef] [PubMed]

44. Srivastava, V.; Gusain, D.; Sharma, Y.C. Synthesis, characterization and application of zinc oxide nanoparticles (n-ZnO). Ceram. Int. 2013, 39, 9803-9808. [CrossRef]

45. de Souza, T.A.J.; Souza, L.R.R.; Franchi, L.P. Silver nanoparticles: An integrated view of green synthesis methods, transformation in the environment, and toxicity. Ecotoxicol. Environ. Saf. 2019, 171, 691-700. [CrossRef]

46. Luo, Y.; Hong, Y.; Shen, L.; Wu, F.; Lin, X. Multifunctional Role of Polyvinylpyrrolidone in Pharmaceutical Formulations. AAPS PharmSciTech 2021, 22, 34. [CrossRef] [PubMed]

47. Fahmy, H.M.; Mosleh, A.M.; Elghany, A.A.; Shams-Eldin, E.; Abu Serea, E.S.; Ali, S.A.; Shalan, A.E. Coated silver nanoparticles: Synthesis, cytotoxicity, and optical properties. RSC Adv. 2019, 9, 20118-20136. [CrossRef]

48. Wan, Y.; Guo, Z.; Jiang, X.; Fang, K.; Lu, X.; Zhang, Y.; Gu, N. Quasi-spherical silver nanoparticles: Aqueous synthesis and size control by the seed-mediated Lee-Meisel method. J. Colloid Interface Sci. 2013, 394, 263-268. [CrossRef]

49. Szerencsés, B.; Igaz, N.; Tóbiás, Á.; Prucsi, Z.; Rónavári, A.; Bélteky, P.; Madarász, D.; Papp, C.; Makra, I.; Vágvölgyi, C.; et al. Size-dependent activity of silver nanoparticles on the morphological switch and biofilm formation of opportunistic pathogenic yeasts. BMC Microbiol. 2020, 20, 13. [CrossRef]

50. Huang, T.; Xu, X.-H.N. Synthesis and characterization of tunable rainbow colored colloidal silver nanoparticles using singlenanoparticle plasmonic microscopy and spectroscopy. J. Mater. Chem. 2010, 20, 9867-9876. [CrossRef]

51. Kararli, T.T. Comparison of the gastrointestinal anatomy, physiology, and biochemistry of humans and commonly used laboratory animals. Biopharm. Drug Dispos. 1995, 16, 351-380. [CrossRef] [PubMed]

52. Eichelberger, L.; Richter, R.B. Water, Nitrogen and Electrolyte Concentration in the Brain. J. Biol. Chem. 1944, 154, 21-29. [CrossRef]

53. Yao, T.; Asayama, Y. Animal-cell culture media: History, characteristics, and current issues. Reprod. Med. Biol. 2017, 16, 99-117. [CrossRef] [PubMed]

54. Freckmann, G.; Hagenlocher, S.; Baumstark, A.; Jendrike, N.; Gillen, R.C.; Rössner, K.; Haug, C. Continuous Glucose Profiles in Healthy Subjects under Everyday Life Conditions and after Different Meals. J. Diabetes Sci. Technol. 2007, 1, 695-703. [CrossRef] [PubMed]

55. Strazzullo, P.; Leclercq, C. Sodium. Adv. Nutr. 2014, 5, 188-190. [CrossRef] [PubMed]

56. Bhattacharjee, S. DLS and zeta potential—What they are and what they are not? J. Control. Release 2016, 235, 337-351. [CrossRef] 